\title{
Évolution du potentiel hydrique foliaire et de la conductance stomatique de quatre chênes méditerranéens lors d'une période de dessèchement
}

\author{
M Acherar ${ }^{1}$, S Rambal $2^{*}$, J Lepart 2 \\ 1 Université de Tizi-Ouzou, institut de biologie, 15000 Tizi-Ouzou, Algérie; \\ ${ }^{2}$ Centre d'écologie fonctionnelle et évolutive Louis Emberger, CNRS, BP 5051, \\ 34033 Montpellier Cedex, France
}

(Reçu le 26 juin 1990; accepté le 3 juin 1991)

\begin{abstract}
Résumé - La réponse écophysiologique à la contrainte hydrique de 2 chênes caducifoliés (Quercus afares Pomel et $Q$ faginea Willd) et de 2 chênes sempervirents ( $Q$ ilex $L$ et $Q$ suber $L$ ) a été étudiée en conditions expérimentales. De jeunes plants, âgés de 2 ans et élevés en pots de $30 \mathrm{I}$, ont été soumis à un cycle de dessèchement au cours duquel nous avons mesuré les potentiels hydriques foliaires de base et minimal ainsi que la conductance stomatique. Pour les 4 chênes étudiés, nos résultats montrent que les évolutions journalières de la conductance stomatique sont conformes aux patrons proposés par Hinckley et al (1978 et 1983). Parallèlement à cette réponse des espèces à la variation à court terme de leurs ressources en eau, le potentiel hydrique de base foliaire contrôle la conductance stomatique maximale. Au cours du dessèchement, la conductance maximale relative $\left(g_{s r \max }\right)$ décroît rapidement et est bien décrite par une fonction homographique de la forme $g_{s r \max }=\left(-0,47+2,61 \psi_{b}\right)^{-1}$ pour les chênes sempervirents et $g_{s r \text { max }}=\left(-1,94+7,39 \psi_{b}\right)^{-1}$ pour les chênes caducifoliés. Ces résultats qui permettent de séparer ces espèces en 2 groupes isofonctionnels, les chênes sempervirents et les chênes caducifoliés, n'expliquent que partiellement leurs aires de répartition. Cependant, ils nous renvoient à des questions plus générales concernant les mécanismes d'utilisation de l'eau par les chênes méditerranéens.
\end{abstract}

Quercus / caducifolié / sempervirent / conductance stomatique / potentiel hydrique / contrainte hydrique / espèce méditerranéenne

Summary - Influence of soil drying on leaf water potential and stomatal conductance in four Mediterranean oak species. The water relations and the responses of 2 evergreen Quercus species (Quercus ilex $L$ and $Q$ suber $L$ ) and 2 deciduous species ( $Q$ afares Pomel and $Q$ faginea Willd) were studied under experimental conditions. Two-yr old seedlings grown in 30-l pots were subjected to a drying period where stomatal conductance, pre-dawn leaf water potential and minimum leaf water potential were measured. The results show that, for all species, the daily course of stomatal conductance agrees with the patterns proposed by Hinckley et al $(1978,1983)$. Concurrent with the species responses to short-term variation in water availability, it was found that pre-dawn leaf water potential controlled the maximum daily leaf conductance. There was a strong correlation between pre-dawn leaf potential and maximum daily conductance $\left(\mathrm{g}_{\mathrm{srmax}}\right)$ as described by the reciprocal function $\mathrm{g}_{\mathrm{sr} \max }=\left(-0.47+2.61 \psi_{\mathrm{b}}\right)^{-1}$ for the evergreen oaks and $\mathrm{g}_{\mathrm{srmax}}=\left(-1.94+7.39 \psi_{\mathrm{b}}\right)^{-1}$ for the deciduous ones. The differences between the 2 groups may partially explain their geographic distribu-

\footnotetext{
* Correspondance et tirés à part
} 
tions, and also lead to general questions concerning the mechanisms of water use in the Mediterranean oak species.

Quercus / deciduous / evergreen / stomatal conductance / water potential / water stress / Mediterranean species

\section{INTRODUCTION}

Le rôle de la sécheresse dans la répartition des espèces du genre Quercus au sein de l'aire isoclimatique méditerranéenne a été souligné depuis longtemps (Emberger, 1930; Braun-Blanquet et al, 1952; Larcher, 1960; Di Castri et Mooney, 1973; Quezel, 1976; Chalabi, 1980).

Pour expliquer la meilleure adaptation de certaines espèces à la sécheresse, Larcher (1960), Specht (1969), Kummerow (1973), Mooney et al (1974), Christodoulakis et Mitrakos (1987) et Romane (1987) font appel à des différences d'ordre morphologique, notamment au niveau foliaire, en se basant sur le concept de sclérophyllie introduit par Schimper (1898). Le rôle de la sclérophyllie dans l'économie de l'eau a été cependant contesté très tôt puisque Kamp (1930), cité par Amireh (1960) et Larcher (1960), observent que la morphologie des feuilles de 2 chênes sclérophylles méditerranéens Quercus coccifera et Quercus ilex n'assure pas une réduction de la transpiration. Salleo et Lo Gullo (1990) aboutissent aux mêmes conclusions en montrant que le degré de sclérophyllie de Quercus ilex et Quercus suber intervient très peu dans l'économie de l'eau. Mooney et al (1974), Miller et Mooney (1976), Rundel (1980), Merino et al (1982) et Merino (1987) suggèrent que les différences phénologiques (opposition entre caractères sempervirent et caducifolié du feuillage) jouent un rôle important dans l'économie de l'eau. Enfin, avec le développement des techniques de me- sures écophysiologiques, des différences dans le fonctionnement physiologique des espèces sont recherchées, notamment au niveau des échanges hydriques (Roberts et Knoerr, 1977; Roberts et al, 1981; Poole et Miller, 1975; Aussenac et Valette, 1982; Guyon, 1987; Vignes, 1988). Par ailleurs, pour Keeley et Zedler (1978), Oechel (1988), Hasting et al (1989), Davis (1989) et Mooney (1989), les différences de traits adaptatifs face à la contrainte hydrique sont à étudier au stade semis. C'est lors de la phase d'installation que l'on observe la mortalité la plus élevée pour de nombreuses espèces ligneuses méditerranéennes.

En Afrique du Nord, les travaux des phytogéographes (Lapie, 1909; Maire, 1926) ou des phytosociologues (Quezel, 1976) mettent en évidence des relations étroites entre la répartition des chênes et les variations altitudinales du climat. Le chêne afarès (Quercus afares Pomel), le chêne zeen (Quercus faginea Willd), qui sont 2 espèces caducifoliées, occupent les étages bioclimatiques humides et subhumides, le chêne vert (Quercus ilex $L$ ) et le chêne liège (Quercus suber $L$ ) qui sont sempervirents, se rencontrent dans les étages subhumides voire semi-arides pour Qilex.

Dans ce travail, nous comparons les réponses écophysiologiques, face à la contrainte hydrique, de jeunes individus de ces 4 espèces cultivés en pots, et étudions dans quelle mesure ces réponses contribuent à expliquer leurs statuts écologiques et leurs aires de répartition. 


\section{MATÉRIELS ET MÉTHODES}

L'expérimentation a été menée au centre Emberger du CNRS de Montpellier, sur des jeunes individus issus de glands récoltés dans des stations typiques de chacune des espèces dans le massif de l'Akfadou $\left(36,48^{\circ} \mathrm{N}\right.$ et $\left.4,30^{\circ} \mathrm{E}\right)$, situé à une centaine de $\mathrm{km}$ à l'est d'Alger. Les individus âgés de 2 ans ont été cultivés en pots de 301 contenant un mélange à parts égales de terre et de terreau. Les pots ont été régulièrement alimentés en eau jusqu'au 7 juillet 1988 , date du début du cycle de dessèchement. Pour supprimer les pertes d'eau par évaporation, un bouchon de polystyrène de $2 \mathrm{~cm}$ d'épaisseur ne laissant passer que la tige est placé à la surface des pots. L'expérience a été arrêtée lorsque des signes de flétrissement apparurent sur les feuilles. Cette situation a été atteinte après 15 j pour $Q$ afares, $Q$ faginea et $Q$ suber. $Q$ ilex ne présente des signes de flétrissement que le 2 août, soit 11 j plus tard.

Les mesures ont été effectuées sur 12 individus ( 3 par espèce) de hauteur comprise entre 0,70 et $0,80 \mathrm{~m}$. La conductance stomatique, la transpiration foliaire, ainsi que le rayonnement photosynthétiquement actif, l'humidité relative de l'air, la température foliaire et celle de l'air, ont été mesurés à l'aide d'un autoporomètre LICOR LI1600 (LICOR Ltd, Lincoln, Nebraska, USA). Les mesures ont porté sur 2 ou 3 feuilles matures et ensoleillées par individu. Ces feuilles choisies au hasard ont été suivies pendant toute

Tableau I. Paramètres climatiques des journées de mesure. $T_{m}$ et $T_{M}$ : température minimale et maximale en ${ }^{\circ} \mathrm{C}$ et $R g$ : rayonnement global journalier en J.cm ${ }^{-2}$.

\begin{tabular}{rccc} 
Date & $T_{m}\left({ }^{\circ} \mathrm{C}\right)$ & $T_{M}\left({ }^{\circ} \mathrm{C}\right)$ & $R g\left(\mathrm{~J} . \mathrm{cm}^{-2}\right)$ \\
\hline 7 juill & 17,0 & 30,0 & 2860 \\
11 juill & 17,0 & 31,5 & 2730 \\
15 juill & 17,5 & 26,5 & 2830 \\
19 juill & 21,5 & 33,0 & 2640 \\
22 juill & 17,0 & 33,0 & 2680 \\
26 juill & 17,0 & 30,0 & 2680 \\
2 août & 18,0 & 28,0 & 1920 \\
\hline
\end{tabular}

la durée de l'expérience. Le potentiel de base $\left(\psi_{b}\right)$ et le potentiel minimum $\left(\psi_{m}\right)$ ont été mesurés respectivement au lever du jour et au midi solaire à l'aide d'une chambre à pression (PMS 1000, Corvallis, Oregon, USA) selon la technique développée par Scholander et al (1965).

Les conditions climatiques des journées de mesure sont résumées dans le tableau I. Les températures minimales de ces journées furent toutes supérieures à $17^{\circ} \mathrm{C}$, les températures maximales fluctuèrent de 26,5 à $33^{\circ} \mathrm{C}$. Le rayonnement global dépassa généralement $2600 \mathrm{~J} . \mathrm{cm}^{-2}$. II n'atteignit cependant que 1920 J.cm-2 le 2 août.

\section{RÉSULTATS}

\section{Potentiels hydriques foliaires}

Les valeurs du potentiel de base $\left(\psi_{b}\right)$ et du potentiel minimal $\left(\psi_{m}\right)$ en début et en fin de dessèchement sont reportées dans le tableau II. Au début du dessèchement, le 7 juillet, les potentiels de base varient entre $-0,20$ et $-0,95 \mathrm{MPa}$. Les chênes sempervirents montrent les $\psi_{b}$ les plus bas $-0,65 \pm 0,11 \mathrm{MPa}$ pour $Q$ ilex et $-0,73 \pm$ $0,16 \mathrm{MPa}$ pour $Q$ suber. Les chênes caducifoliés ont des valeurs plus élevées $-0,25$ $\pm 0,04 \mathrm{MPa}$ pour $Q$ afares et $-0,23 \pm 0,05$ $\mathrm{MPa}$ pour $Q$ faginea. Cette dichotomie entre chênes sempervirents et caducifoliés n'apparaît plus pour les potentiels minima. $Q$ ilex et $Q$ afares présentent les potentiels les plus bas, $-3,15 \pm 0,50 \mathrm{MPa}$ et $-3,25 \pm$ $0,10 \mathrm{MPa}$ respectivement. En fin de dessèchement, le 22 juillet, le potentiel de base varie entre $-1,35 \pm 0,18 \mathrm{MPa}$ pour $Q$ ilex et $-3,70 \pm 1,37 \mathrm{MPa}$ pour $Q$ suber qui présente le potentiel le plus bas. On observe une grande variabilité entre individus d'une même espèce. Cette variabilité traduit, en première approximation, des différences de consommation en eau, déterminées en grande partie par les surfaces foliaires des 
Tableau II. Comparaison des potentiels hydriques foliaires de base $\psi_{b}$ et minimal $\psi_{m}$, en $-\mathrm{MPa}$, et des conductances stomatiques maximales, en mmol. $\mathrm{m}^{-2} \cdot \mathrm{s}^{-1}$, atteints en début (1) et en fin de déssèchement (2), les 7 et 22 juillet respectivement. Les surfaces foliaires des individus sont exprimées en $\mathrm{m}^{2}$ de feuille.

\begin{tabular}{|c|c|c|c|c|c|c|c|c|}
\hline \multirow[t]{2}{*}{ Espèce } & \multirow[t]{2}{*}{ Individu } & \multirow{2}{*}{$\begin{array}{l}\text { Surface } \\
\text { foliaire }\end{array}$} & \multicolumn{2}{|c|}{$\psi_{b}$} & \multicolumn{2}{|c|}{$\psi_{m}$} & \multicolumn{2}{|c|}{$g_{\text {smax }}$} \\
\hline & & & 1 & 2 & 1 & 2 & 1 & 2 \\
\hline Quercus ilex & $\begin{array}{l}\text { Q14 } \\
\text { Q117 } \\
\text { Q18 }\end{array}$ & $\begin{array}{l}0,13 \\
0,12 \\
0,28\end{array}$ & $\begin{array}{l}0,75 \\
0,50 \\
0,65\end{array}$ & $\begin{array}{l}1,00 \\
0,80 \\
1,15\end{array}$ & $\begin{array}{l}3,65 \\
3,20 \\
2,60\end{array}$ & $\begin{array}{l}3,25 \\
2,40 \\
3,05\end{array}$ & $\begin{array}{l}376 \\
304 \\
362\end{array}$ & $\begin{array}{r}95 \\
210 \\
76\end{array}$ \\
\hline Quercus suber & $\begin{array}{l}\text { QS1 } \\
\text { QS7 } \\
\text { QS8 }\end{array}$ & $\begin{array}{l}0,86 \\
0,68 \\
0,72\end{array}$ & $\begin{array}{l}0,70 \\
0,95 \\
0,55\end{array}$ & $\begin{array}{l}5,20 \\
3,40 \\
2,50\end{array}$ & $\begin{array}{l}2,25 \\
2,50 \\
2,95\end{array}$ & $\begin{array}{l}6,50 \\
3,65 \\
3,45\end{array}$ & $\begin{array}{l}213 \\
254 \\
127\end{array}$ & $\begin{array}{l}12 \\
47 \\
34\end{array}$ \\
\hline Quercus afares & $\begin{array}{l}\text { QA1 } \\
\text { QA7 } \\
\text { QA9 }\end{array}$ & $\begin{array}{l}0,50 \\
0,27 \\
0,48\end{array}$ & $\begin{array}{l}0,20 \\
0,25 \\
0,30\end{array}$ & $\begin{array}{l}2,30 \\
1,75 \\
4,05\end{array}$ & $\begin{array}{l}3,25 \\
3,15 \\
3,35\end{array}$ & $\begin{array}{l}3,30 \\
3,35 \\
5,65\end{array}$ & $\begin{array}{l}395 \\
410 \\
539\end{array}$ & $\begin{array}{l}34 \\
44 \\
20\end{array}$ \\
\hline Quercus faginea & $\begin{array}{ll}\text { QF2 } \\
\text { QF6 } \\
\text { QF10 }\end{array}$ & $\begin{array}{l}0,37 \\
0,61 \\
0,25\end{array}$ & $\begin{array}{l}0,20 \\
0,20 \\
0,30\end{array}$ & $\begin{array}{l}3,10 \\
3,10 \\
4,20\end{array}$ & $\begin{array}{l}2,25 \\
2,25 \\
1,85\end{array}$ & $\begin{array}{l}3,45 \\
3,45 \\
4,90\end{array}$ & $\begin{array}{l}351 \\
351 \\
289\end{array}$ & $\begin{array}{l}16 \\
16 \\
10\end{array}$ \\
\hline
\end{tabular}

individus. L'analyse de covariance de $\psi_{b}$ montre que le facteur "espèce" n'est pas significatif alors que le cofacteur "surface foliaire» est significatif au seuil de $5 \%$.

Sur la figure 1, l'évolution de $\psi_{b}$ est présentée pour 2 groupes d'individus de surfaces foliaires proches. Pour les individus ayant des surfaces foliaires voisines de $0,25 \mathrm{~cm}^{2}$ (tableau II), le $\psi_{b}$ de $Q$ faginea (QF10) chute rapidement de $-0,30 \mathrm{MPa}$ pour atteindre $-4,20 \mathrm{MPa}$ le 22 juillet (fig 1), alors que celui de $Q$ ilex (Q18), diminue relativement peu, puisqu'il passe de $-0,65$ à $-1,15 \mathrm{MPa}$; la décroissance du $\psi_{b}$ de $Q$ afares (QA1) est intermédiaire. Pour le chêne zeen QF6 et le chêne liège QS7, dont les surfaces sont proches de 0,65 $\mathrm{cm}^{2}$, les potentiels de base décroissent de la même manière pour atteindre des va-

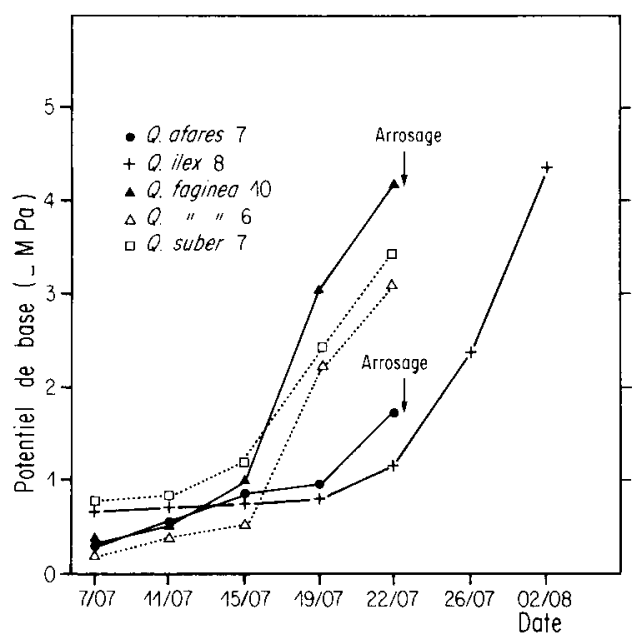

Fig 1. Évolution du potentiel de base de quelques individus au cours du dessèchement. 
leurs relativement proches, $-2,25$ et $-2,5$ MPa respectivement.

\section{Conductance stomatique}

\section{Évolution journalière}

Quelques courbes d'évolution journalière de la conductance stomatique au cours du dessèchement sont représentées sur la figure 2. De manière générale, au début du dessèchement, les courbes d'évolution présentent une forme en cloche avec un maximum en milieu de journée, puis s'aplatissent au fur et à mesure que le potentiel de base diminue. Pour comparer ces courbes journalières, nous les avons classées selon 3 patrons d'évolution en utilisant la typologie de Hinckley et al (1978 et 1983).

Les courbes de type 1 sont en forme de cloche avec un maximum en milieu de journée. Elles correspondent à des situations dans lesquelles le potentiel hydrique du sol, le potentiel hydrique foliaire, et le déficit de pression de vapeur saturante de l'air ne semblent pas limiter la conductance stomatique. Ce type n'est rencontré, dans notre cas, qu'au début de l'expérience lorsque la teneur en eau du sol des pots se trouve au voisinage de la capacité au champ.

Les courbes de type 2 présentent 2 maxima, l'un en début de journée, l'autre l'après-midi, séparés par une dépression en milieu de journée. Elles correspondent à des situations pour lesquelles un ou plusieurs des facteurs précédemment évoqués limitent la conductance stomatique.

Dans le type 3 , les courbes présentent seulement un maximum en début de journée. La plante atteindrait un niveau de potentiel hydrique foliaire à partir duquel, selon Hinckley et al, il y aurait perte de turgescence des feuilles.

Sur la figure 2, le type 2 est peu représenté. Le passage du type 1 au type 3 se fait rapidement au cours du dessèchement. On observe généralement une dissymétrie des courbes. Les plus fortes conductances sont obtenues en début de matinée. Nous avons représenté pour chaque individu, dans le tableau III, la durée
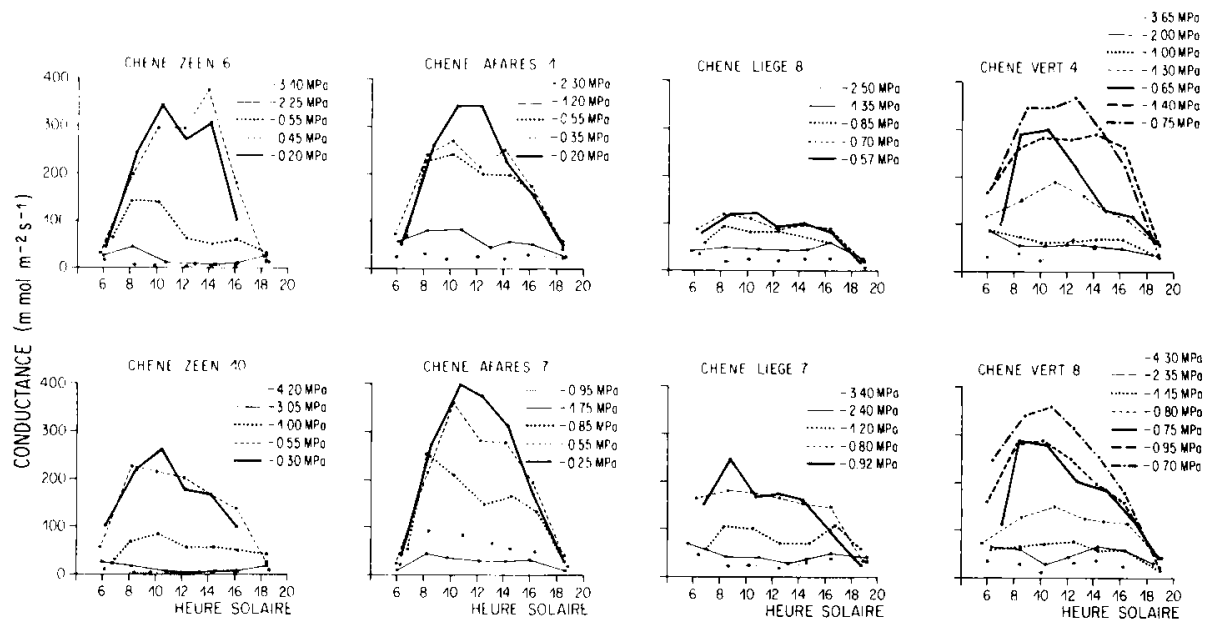

Fig 2. Évolution journalière de la conductance stomatique de quelques individus au cours du dessèchement. Les chiffres indiquent les potentiels de base des journées correspondantes. 
Tableau III. Durée du déssèchement, en jours, nécessaire pour atteindre une évolution journalière de la conductance stomatique de type 3 . $\psi_{b}$ et $\psi_{m}$ représentent les potentiels hydriques foliaires de base et minimal, en -MPa, atteints au cours de la même journée.

\begin{tabular}{llccc}
\hline Espèce & Individu & $\begin{array}{c}\text { Durée } \\
\text { (j) }\end{array}$ & $\psi_{b}$ & $\psi_{m}$ \\
& & & & \\
\hline \multirow{4}{*}{ Quercus ilex } & Q14 & 20 & 2,0 & 3,5 \\
& Q117 & 27 & 2,6 & 4,4 \\
& Q18 & 20 & 2,3 & 4,1 \\
Quercus suber & QS1 & 9 & 2,4 & 3,5 \\
& QS7 & 13 & 2,4 & 2,8 \\
& QS8 & 16 & 2,5 & 3,5 \\
Quercus afares & QA1 & 16 & 2,3 & 3,3 \\
& QA7 & 16 & 1,8 & 3,4 \\
& QA9 & 13 & 2,6 & 4,5 \\
& & & & \\
Quercus faginea & QF2 & 13 & 1,3 & 2,2 \\
& QF6 & 13 & 2,3 & 2,8 \\
& QF10 & 13 & 3,1 & 3,7 \\
& & & & \\
\hline
\end{tabular}

de dessèchement nécessaire pour que la conductance stomatique soit de type 3 ainsi que les potentiels hydriques de base et minimal correspondant. Pour les individus Q18, QA7 et QF10, de surfaces foliaires proches, $0,28,0,27$ et $0,25 \mathrm{~m}^{2}$ respectivement, c'est $Q$ faginea qui atteint le plus rapidement le type 3 suivi par $Q$ afares et par $Q$ ilex. Pour QS7 et QF6 de surfaces foliaires 0,68 et $0,61 \mathrm{~m}^{2}$, le passage à un fonctionnement de type 3 a lieu après 13 j de dessèchement. Les potentiels de base atteints sont relativement proches et s'échelonnent entre -2,2 $\mathrm{MPa}$ pour $Q$ faginea et $-2,4 \mathrm{MPa}$ pour $Q$ ilex, avec une grande variabilité pour les chênes caducifoliés, notamment pour $Q$ faginea. Cette importante variabilité est la conséquence de la nature discontinue de nos observations (tous les $4 j$ environ) et de la rapidité du phénomène.

\section{Conductance maximale}

Au début du dessèchement, les conductances maximales observées s'échelonnent entre $198 \pm 53 \mathrm{mmol} . \mathrm{m}^{-2} \cdot \mathrm{s}^{-1}$ pour $Q$ suber et $448 \pm 65 \mathrm{mmol} \cdot \mathrm{m}^{-2} \cdot \mathrm{s}^{-1}$ pour $Q$ afares (tableau II). $Q$ ilex et $Q$ faginea ont des valeurs relativement proches, $347 \pm 31$ et $339 \pm 37 \mathrm{mmol} . \mathrm{m}^{-2} . \mathrm{s}^{-1}$ respectivement. Parmi les chênes sempervirents, $Q$ ilex montre, dans les conditions de notre expérience, une conductance maximale moyenne plus élevée que celle de $Q$ suber. Nous observons par ailleurs une assez forte variabilité interindividuelle au sein de chaque espèce, sauf chez $Q$ ilex. En fin de dessèchement, le 22 juillet, les conductances maximales des chênes caducifoliés sont faibles, $20 \pm 13$ $\mathrm{mmol} \cdot \mathrm{m}^{-2} \cdot \mathrm{s}^{-1}$ pour $Q$ faginea et $33 \pm 10$ $\mathrm{mmol} \cdot \mathrm{m}^{-2} \cdot \mathrm{s}^{-1}$ pour $Q$ afares, de même que celles de $Q$ suber, $31 \pm 15$ mmol. $\mathrm{m}^{-2} \cdot \mathrm{s}^{-1}$. Elles se maintiennent à des valeurs élevées pour $Q$ ilex, $127 \pm 59$ $\mathrm{mmol} . \mathrm{m}^{-2} \cdot \mathrm{s}^{-1}$.

La figure 3 présente les changements de la conductance stomatique maximale $\left(g_{\text {smax }}\right)$ en fonction du potentiel de base pour les 4 espèces. Ces relations ont été ajustées à des équations de la forme $g_{s \max }$ $=\left(a+b \psi_{b}\right)^{-1}$ dont les paramètres d'ajustement sont présentés dans le tableau $\mathrm{V}$. Cette décroissance de la conductance maximale est bien décrite par cette fonction réciproque puisque les coefficients de détermination $\left(r^{2}\right)$ s'échelonnent entre 0,81 et 0,89 .

Les changements de la conductance maximale relative $\left(g_{s m \text { max }}\right)$, c'est-à-dire le rapport de la conductance maximale $g_{\text {smax }}$ et de sa valeur maximale estimée en début de dessèchement, en fonction du potentiel 

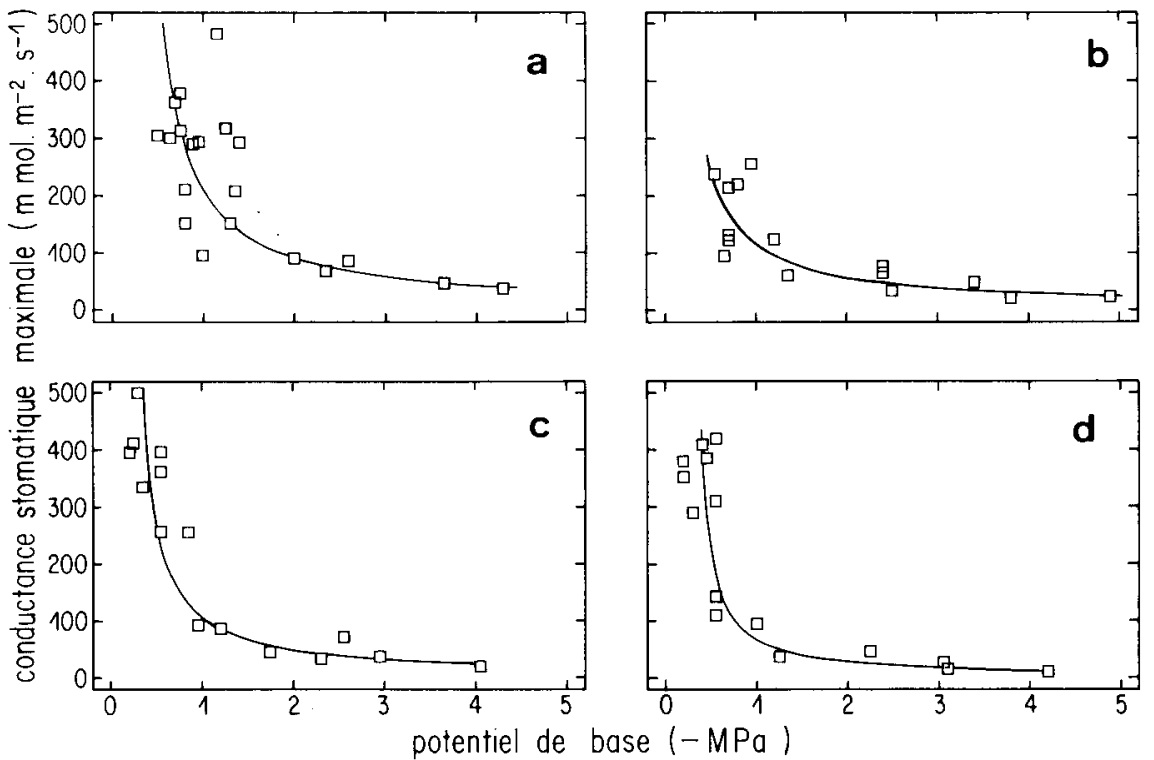

Fig 3. Relation entre la conductance maximale et le potentiel de base pour a) Quercus ilex, b) Quercus suber, c) Quercus afares et d) Quercus faginea.

de base suivent des équations de la même forme $g_{s \max }=\left(a+b \psi_{b}\right)^{-1}$ (tableau IV). Nous avons estimé, en extrapolant vers des $\psi_{b}$ voisins de 0 , les valeurs maximales à $500,430,420$ et $280 \mathrm{mmol} . \mathrm{m}^{-2} . \mathrm{s}^{-1}$ pour $Q$ afares, $Q$ faginea, $Q$ ilex et $Q$ suber

Tableau IV. Paramètres des équations de régressions entre d'une part, la conductance maximale $g_{s m a x}$, en mmol.m $\mathrm{m}^{-2} \cdot \mathrm{s}^{-1}$, et le potentiel de base $\psi_{b}$, en MPa, $\mathrm{g}_{\text {smax }}=1 /\left(a+b \psi_{b}\right)$ et d'autre part, entre la conductance maximale relative $g_{s m a x}$, et le potentiel de base $\psi_{b}$, en $\mathrm{MPa}, \mathrm{g}_{s \max }=1 /\left(a+b \psi_{b}\right)$; $r^{2}$ est le coefficient de détermination; $n$ est le nombre d'observations.

$b$

$r^{2}$

$n$

\section{Conductance maximale}

Quercus ilex

Quercus suber

Quercus afares

Quercus faginea

$$
\begin{aligned}
& -1,50 \times 10^{-3} \\
& -6,20 \times 10^{-4} \\
& -1,96 \times 10^{-3} \\
& -5,88 \times 10^{-3}
\end{aligned}
$$

$6,18 \times 10^{-3}$

$9,21 \times 10^{-3}$

$1,13 \times 10^{-3}$

$2,07 \times 10^{-2}$
0,88

0,81

0,89

0,89
20

15

15

15

Conductance maximale relative

Chênes sempervirents

Chênes caducifoliés
$-0,473$
$-1,94$ 
respectivement. Ces évolutions sont significativement différentes, au seuil de $5 \%$, pour les chênes sempervirents et caducifoliés, mais ne diffèrent pas entre $Q$ ilex et $Q$ suber d'une part et $Q$ afares et $Q$ faginea d'autre part. La comparaison de ces courbes a été réalisée par le test de Tukey-Kramer (Sokal et Rohlf, 1981). De ce fait, nous n'avons représenté sur la figure 4 que 2 ajustements, l'un pour les chênes caducifoliés et l'autre pour les chênes sempervirents. Les conductances maximales des chênes caducifoliés chutent plus rapidement que celles des chênes sempervirents. Chez les chênes caducifoliés, lorsque le potentiel de base est de $-1 \mathrm{MPa}$, la conductance stomatique n'est plus que de $18 \%$ de la conductance maximale. Pour un $\psi_{b}$ de $-2 \mathrm{MPa}$, elle est pratiquement nulle $(8 \%)$. Chez les chênes sempervirents, elle passe de 47 à $21 \%$ lorsque $\psi_{b}$ passe de -1 à $-2 \mathrm{MPa}$. Elle n'atteint des valeurs pratiquement nulles (8\%) que vers $-5 \mathrm{MPa}$.

\section{DISCUSSION ET CONCLUSIONS}

En début de dessèchement, lorsque le sol est à la capacité au champ, les potentiels de base des chênes sempervirents présentent des valeurs plus basses que celles des chênes caducifoliés. Cette différence entre espèces caducifoliées et sempervirentes a été également observée in situ sous climat méditerranéen (Griffin, 1973; Aussenac et Valette, 1982), tempéré (Aussenac et Granier, 1978; Bowman et Roberts, 1985) et tropical (Sobrado, 1986; Fanjul et Barradas, 1987). Elle est due, selon Roberts et Knoerr (1977), Hinckley et al (1980) et Bowman et Roberts (1985), à des différences entre les composantes du potentiel hydrique foliaire, potentiels de turgescence et osmotique. Elle pourrait aussi résulter d'une transpiration nocturne provoquée par un fort déficit de pression de vapeur saturante en période estivale.

Au cours du dessèchement, l'équilibre entre le sol et les racines se fait à des po-

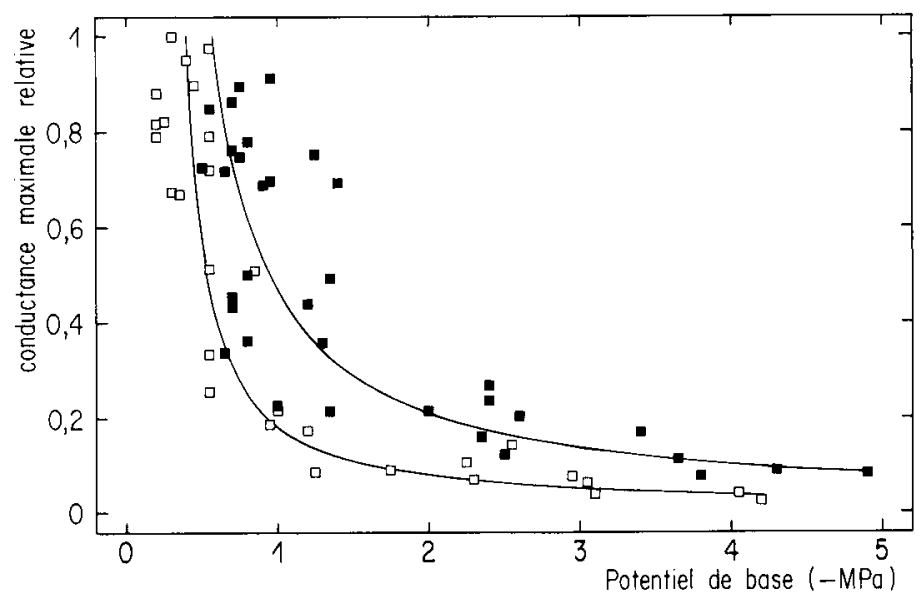

Fig 4. Relation entre la conductance maximale relative et le potentiel de base pour les chênes sempervirents et les chênes caducifoliés. La conductance est exprimée en pourcentage de la conductance maximale estimée pour des potentiels de base voisins de 0 . 
tentiels de plus en plus négatifs. Pour des individus de surfaces foliaires proches, cette décroissance est plus rapide pour $Q$ faginea que pour $Q$ ilex et $Q$ afares. Cela peut être, soit lié à une très forte transpiration de $Q$ faginea entraînant un dessèchement rapide du sol au voisinage des racines, soit la conséquence d'une exploitation superficielle du sol.

Tenhunen et al (1981 et 1982) et Lange et al (1982) observent une dépression de la conductance stomatique au midi solaire chez de nombreuses espèces ligneuses méditerranéennes, notamment $Q$ coccifera, $Q$ ilex et $Q$ suber. Cette dépression est considérée par ces auteurs comme une caractéristique des espèces méditerranéennes qui leur permet de limiter les pertes en eau au moment de la journée où la demande climatique est la plus forte. Elle serait contrôlée par le déficit de pression de vapeur saturante de l'air. Par contre, selon Hinckley et al (1983), elle dépendrait de la conjugaison de plusieurs facteurs, notamment du potentiel hydrique instantané du végétal et, selon Correia et al (1990), elle serait le résultat d'effets inhibiteurs des forts rayonnements photosynthétiques sur les chloroplastes. Les dépressions au midi solaire sont peu marquées dans l'expérimentation que nous avons réalisée. C'est aussi le cas des observations faites in situ sur des arbres adultes de $Q$ ilex, en Espagne, par Sala Serra et al (1990) et de $Q$ suber, au Portugal, par Oliveira et al (1991), qui mettent aussi en évidence la faible amplitude de ce phénomène.

Nous observons que les valeurs de conductance maximale des chênes sempervirents sont aussi élevées que celles des chênes caducifoliés. Nos valeurs pour $Q$ suber sont plus basses que celles de Tenhunen et al (1987) et de Oliveira et al (1991), 370 et $340 \mathrm{mmol} \cdot \mathrm{m}^{-2} \cdot \mathrm{s}^{-1}$ respectivement. Elles ont été obtenues à des po- tentiels de base voisins de $-0,3 \mathrm{MPa}$. Pour $Q$ ilex, elles atteignent $280 \mathrm{mmol} \cdot \mathrm{m}^{-2} . \mathrm{s}^{-1}$ chez Rhizopoulos et al (1990) et 260 mmol.m-2.s $\mathrm{s}^{-1}$ chez Sala Serra et al (1990). Toutefois, il ne faut accorder à ces comparaisons qu'une valeur relative car ces travaux sont conduits dans des conditions de milieu et sur des matériels végétaux (âge des individus, âge des feuilles) différents et pour des potentiels hydriques foliaires plus ou moins proches de 0 .

Pour les 4 chênes étudiés, nos résultats montrent des évolutions journalières de la conductance stomatique tout à fait conformes aux patrons proposés par Hinckley et al (1978 et 1983). Au cours du dessèchement, nous avons noté une décroissance de la conductance maximale qui est bien décrite par une fonction réciproque du potentiel de base de la forme $g_{s r \max }=(\mathrm{a}+$ $\left.b \psi_{b}\right)^{-1}$. Une relation du même type est obtenue par Pereira et al (1987) sur Eucalyptus globulus Labill. Cette décroissance est également observée in situ chez d'autres chênes par Reich et Hinckley (1989) et chez $Q$ ilex par Sala Serra et al (1990). Cependant, chez ces derniers, cette décroissance est décrite par une relation linéaire. Cette différence pourrait être la conséquence d'un dessèchement plus rapide des jeunes individus en pot que celui des individus adultes en conditions naturelles. Toutefois, pour $Q$ ilex et pour des potentiels de base inférieurs à $-1 \mathrm{MPa}$, il n'y a pas de différences significatives entre nos observations et celles de Sala Serra et al (1990). Sur $Q$ suber, nos observations sont pratiquement identiques à celles réalisées par Tenhunen et al (1987) sur des individus adultes.

La décroissance de la conductance maximale, significativement plus rapide pour les chênes caducifoliées que pour les chênes sempervirents, met en évidence une plus grande sensibilité des chênes caducifoliés à la sécheresse édaphique. Ils 
ferment rapidement leurs stomates alors que les chênes sempervirents les maintiennent ouverts à des niveaux plus élevés de contrainte hydrique. Hinckley et al (1983) aboutissent également aux mêmes résultats pour un autre chêne caducifolié méditerranéen, Quercus pubescens, qui présente des signes de flétrissement importants à des potentiels foliaires de $-4,55$ $\mathrm{MPa}$. En terme de résistance à la sécheresse, les chênes caducifoliés évitent le dessèchement en fermant rapidement leurs stomates au détriment de la photosynthèse (Acherar, non publié), alors que les chênes sempervirents maintiennent leurs stomates plus longtemps ouverts, ce qui leur confère une activité photosynthétique plus soutenue à de faibles potentiels hydriques (Acherar, non publié; voir aussi Tenhunen et al, 1985). Cette conductance élevée à faible potentiel de base s'observe également chez d'autres chênes sclérophylles : $Q$ dumosa a une conductance maximale de $80 \mathrm{mmol} \cdot \mathrm{m}^{-2} . \mathrm{s}^{-1}$ à $-3,3 \mathrm{MPa}$ (Hastings et al, 1989); $Q$ ilex, 120 mmol.m-2.s $\mathrm{s}^{-1}$ à $-3,2 \mathrm{MPa}$ (Rambal, non publié); $Q$ coccifera, $150 \mathrm{mmol} \cdot \mathrm{m}^{-2} \cdot \mathrm{s}^{-1}$ à -3 MPa et $Q$ suber, $100-120 \mathrm{mmol} \cdot \mathrm{m}^{-2} . \mathrm{s}^{-1}$ à $-3 \mathrm{MPa}$ (Tenhunen et al, 1981 et 1987).

L'évolution journalière de la conductance stomatique apparaît, à travers les travaux théoriques de Cowan et Farquhar (1977) ou les expérimentations réalisées par William (1983) sur des espèces méditerranéennes du chaparral californien, comme un moyen d'optimiser l'utilisation journalière de l'eau pour l'assimilation du carbone dans un environnement microclimatique fluctuant. Parallèlement à cette réponse du végétal à la variabilité à court terme de ses ressources en eau, le potentiel hydrique foliaire contrôle la conductance stomatique maximale (fig 3). La dynamique de ce contrôle permet de séparer les espèces en 2 groupes isofonctionnels : les chênes sempervirents et les chênes caducifoliés. Cette séparation n'explique que très partiellement leurs aires de répartition géographique qui dépendent principalement des facteurs de mortalité affectant les plantules (voir à ce propos l'étude de Neilson et Wullstein (1985) sur la répartition de 2 chênes nordaméricains, l'un sempervirent, Quercus turbinella, l'autre caducifolié, Quercus gambe(ii). Toutefois, cette séparation renvoie à des questions plus larges concernant les chênes méditerranéens et qui sont, en réalité, une formulation particulière de celles traditionnellement posées lorsque l'on compare les espèces caducifoliées et sempervirentes du chaparral californien (Miller et Mooney, 1976).

Ainsi, les chênes sempervirents, pour profiter de l'avantage d'une ouverture des stomates à des potentiels hydriques foliaires fortement négatifs et le maintien déjà souligné plus haut d'un bilan carboné positif, doivent conserver un flux de transpiration relativement important lors des périodes de sécheresse. Quels traits biologiques (enracinement profond, surface foliaire limitée...) ou combinaison de traits, permettent une gestion saisonnière conservatrice de la ressource en eau ? Par contre, les chênes caducifoliés doivent, dans un temps d'autant plus limité que la contrainte hydrique provoque une fermeture précoce de leurs stomates, assimiler une quantité de carbone au moins égale à celle nécessaire à l'entretien des structures et au renouvellement du feuillage (Merino et al, 1982; Merino, 1987; Mooney, 1989). Une capacité photosynthétique élevée et un faible coût de construction des feuilles suffisent-ils pour assurer leur compétitivité face aux autres espèces ?

En d'autres termes, il s'agit de savoir comment les chênes caducifoliés optimisent l'utilisation de leurs ressources hydriques pendant les seules périodes favorables et comment les chênes 
sempervirents gèrent les ressources en eau en permettant l'assimilation du carbone tout au long de l'année.

\section{REMERCIEMENTS}

Ce travail a été réalisé dans le cadre d'un accord de coopération CNRS-DPRS.

\section{RÉFÉRENCES}

Amireh EH (1960) Le régime hydrique de Quercus coccifera. Thèse de Doctorat de $3^{e}$ cycle, Université de Montpellier

Aussenac G, Granier A (1978) Quelques résultats de cinétique journalière du potentiel de sève des arbres forestiers. Ann Sci For 35 , 19-32

Aussenac G, Valette JC (1982) Comportement hydrique estival de Cedrus atlantica Manetti, Quercus ilex $\mathrm{L}$ et Quercus pubescens Willd et de divers pins dans le Mont Ventoux. Ann Sci For 39, 41-62

Boudy P (1948) Economie forestière nordafricaine. Tome 1, Édition Larose, Paris, $686 p$

Bowman WD, Roberts SW (1985) Seasonal and diurnal water relations adjustments in three evergreen chaparral shrubs. Ecology 66, 738-742

Braun-Blanquet J, Roussine N, Negre R (1952) Les groupements végétaux de la France méditerranéenne. CNRS, service de la carte des groupements végétaux, $297 p$

Chalabi MN (1980) Analyse phytosociologique, phytoécologique, dendrométrique et dendroclimatologique des forêts de Quercus cerris $L$ subsp pseudocerris et contribution à l'étude du genre Quercus en Syrie. Thèse de Doctorat ès sciences, Univ Aix Marseille, $342 p$

Christodoulakis NS, Mitrakos KA (1987) Structural analysis of sclerophylly in eleven evergreen phanerophytes in Greece. In: Plant responses to stress. Functional analysis in mediterranean ecosystems (JD Tenhunen et al, eds), NATO, ASI Ser, Springer Verlag, Berlin, vol G15, 547-552
Correia MJ, Chaves MJ, Pereira JS (1990) Afternoon depression in photosynthesis in grapevine leaves. Evidence for a high light stress effect. J Exp Bot 41, 417-426

Cowan IR, Farquhar GD (1977) Stomatal function in relation to leaf metabolism and environment. Symp Soc Exp Biol 31, 471-505

Davis SD (1989) Patterns in mixed chaparral stands. Differential water status and seedling survival during summer drought. In: The California chaparral. Paradigms reexamined (Keeley SC, ed) Natural History of Los Angeles County, Sciences series $n^{\circ}$ 34, 97-105

Davis SD, Mooney HA (1985) Comparative water relations of adjacent California shrub and grassland communities. Oecologia 66, 522-529

Di Castri F, Mooney HA (éds) (1973) Mediterranean type ecosystems: origin and structure. Springer Verlag, Heidelberg, New York

Emberger $L$ (1930) La végétation de la région méditerranéenne. Essai d'une classification des groupements végétaux. Rev Gén Bot 42, 641-662

Fanjul L, Barradas VL (1987) Diurnal and seasonal variation in the water relations of some deciduous and evergreen trees of a deciduous dry forest of the western coast of Mexico. J Appl Ecol 24, 289-303

Griffin JR (1973) Xylem sap tension in three woodland oaks of central California. Ecology $54,152-159$

Guyon JP (1987) Analyse des courbes de "pression-volume" de rameaux de trois espèces forestières. Acta Oecol Oecol Applic 8, 363-370

Hastings SJ, Oechel WC, Sionit N (1989) Water relations and photosynthesis of chaparral resprouts and seedlings following fire and hand clearing. In: The California chaparral. Paradigms reexamined (Keeley SC, ed) Natural history of Los Angeles County. Sciences series $n^{\circ} 34,107-113$

Hinckley TM, Duhme F, Hinckley AR, Richter H (1980) Water relations of drought hardy shrubs : osmotic potential and stomatal reactivity. Plant Cell Environ 3, 131-140

Hinckley TM, Duhme F, Hinckley AR, Richter $H$ (1983) Drought relations of shrub species: assessment of the mecanism of drought resistance. Oecologia 59, 344-350 
Hinckley TM, Lassoie JP, Running SW (1978) Temporal and spatial variations in the water status of forest trees. For Sci Monogr 20, 172

Keeley JE, Zedler PH (1978) Reproduction of chaparral shrubs after fire: a comparison of sprouting and seeding strategies. Am Midl Nat $99,142-161$

Kummerow $J$ (1973) Comparative anatomy of sclerophylls of mediterranean climatic areas. In: Mediterranean-type ecosystems. Origin and structure (F di Castri, HA Mooney, eds) Springer Verlag, Berlin, Heidelberg, New York, 157-167

Lange OL, Tenhunen JD, Braun M (1982) Midday stomatal closure in mediterranean type sclerophylis under simulated habitat conditions in an environmental chamber. I. Comparison of the behavior of various mediterranean species. Flora 172, 563-579

Lapie G (1909) Étude phytogéographique de la Kabylie du Djurdjura. Thèse de doctorat ès sciences naturelles. Faculté des sciences d'Alger, Delagrave, Paris

Larcher W (1960) Transpiration and photosynthesis of detached leaves and shoots of Quercus pubescens and Quercus ilex during dessiccation under standard conditions. Bull Res Counc Israel 8D, 213-224

Maire (1926) Note phytogéographique de l'Algérie et de la Tunisie avec une carte. Alger

Merino $\mathrm{J}$ (1987) The costs of growing and maintaining leaves of mediterranean plants. In: Plant responses to stress. Functional analysis in mediterranean ecosystems (JD Tenhunen et al, éds), NATO, ASI Series, vol G15, Springer Verlag, Berlin, 553-564

Merino J, Field C, Mooney HA (1982) Construction and maintenance costs of mediterranean-climate evergreen and deciduous leaves. I. Growth and $\mathrm{CO}_{2}$ exchange analysis. Oecologia 53, 208-213

Miller PC, Mooney HA (1976) The origin and structure of American arid zone ecosystems. The producers: interactions between environment, form, and function. In: Critical evaluation of ecosystems analysis in ecosystems research and management (GW Arnold, CT de Wit, éds) Pudoc, Wageningen, 38-56

Mooney HA (1989) Chaparral physiological ecology - Paradigms revisited. In : the California chaparral. Paradigms reexamined (Keeley
SC, éd) Natural History of Los Angeles County. Sciences series $n^{\circ} 34,85-90$

Mooney HA, Parsons DJ, Kummerow J (1974) Plant development in mediterranean climates. In: Phenology and seasonality modeling ( $H$ Lieth, ed), Springer Verlag, Berlin, Heidelberg, New York, 255-267

Neilson RP, Wullstein LH (1985) Comparative drought physiology and biogeography of Quercus gambelii and Quercus turbinella. Am Mid Nat 114, 259-271

Oechel WC (1988) Minimum non-lethal water potentials in mediterranean shrub seedlings. In: Time scales and water stress. Proceedings of 5th international conference on mediterranean ecosystems, 15-21 July 1987, Montpellier, France (Di Castri et al, eds), IUBS Paris, 125-132

Oliveira G, Correia OA, Martins-Louçao MA, Catarino FM (1991) Water relations of cork-oak (Quercus suber $L$ ) under natural conditions. Vegetatio (à paraître)

Pereira JS, Tenhunen JD, Lange OL (1987) Stomatal control of photosynthesis of Eucalyptus globulus Labill trees under field conditions in Portugal. J Exp Bot 38, 1678-1688

Poole DK, Miller PC (1975) Water relations of selected species of chaparral and coastal sage communities. Ecology 56, 1118-1128

Quezel P (1976) Les forêts du pourtour méditerranéen : écologie, conservation et aménagement. In : Forêt et maquis méditerranéens. Note tech. MAB2, UNESCO 9-34

Reich PB, Hinckley TM (1989) Influence of predawn water potential and soil-to-leaf hydraulic conductance on maximum daily leaf diffusive conductance in two oak species. Funct Ecol 3, 719-726

Rhizopoulos S, Mitrakos K (1990) Water relations of evergreen sclerophylls. I. Seasonal changes in the water relations of eleven species from the same environment. Ann Bot 65, 171-178

Roberts SW, Knoerr KR (1977) Components of water potential estimated from xylem pressure measurements in five tree species. Oecologia 28, 191-202

Roberts SW, Miller PC, Valamanesh A (1981) Comparative water relations of four cooccurring chaparral shrub species. Oecologia $48,360-363$ 
Romane F (1987) Efficacité de la distribution des formes de croissance des végétaux pour l'analyse de la végétation à l'échelle régionale. Cas de quelques taillis de chêne vert du Languedoc. Thèse de doctorat ès sciences, univ Aix Marseille, $201 \mathrm{p}$

Rundel PW (1980) Adaptation of MediterraneanClimate Oaks to environmental stress. In: Proceedings of the symposium on the Ecology, management, and utilisation of California oaks. Claremont, CA, 43-54

Sala Serra A, Burriel JA, Tenhunen JD (1990) Spatial and temporal controls on transpiration within a watershed dominated by Quercus ilex. In: International workshop Quercus ilex ecosystems: function, dynamics and management. Montpellier and Barcelona, 17-21 September, abstracts, 89-92

Salleo S, Lo Gullo MA (1990) Sclerophylly and plant water relations in three mediterranean Quercus species. Ann Bot 65, 259-270

Schimper AFW (1898) Pflanzengeographie auf physiologisher grundlage. Gustav Fischer Verlag, Jena

Scholander PF, Hammel HT, Bradstreet ED, Hemmingsen A (1965) Sap pressure in vascular plants. Science 148, 339-346

Sobrado MA (1986) Aspects of tissue water relations and seasonal changes of leaf water potential components of evergreen and deciduous species coexisting in tropical dry forests. Oecologia 68, 413-416

Sokal RR, Rohlf FJ (1981) Biometry. WH Freeman, San Francisco, CA
Specht RL (1969) A comparison of the sclerophyllous vegetation characteristic of Mediterranean type climates in France, California, and southern Australia. I. Structure, morphology and succession. Aust J Bot 17, 277-292

Tenhunen JD, Catarino FM, Lange OL, Oechel WC (eds) (1987) Plant responses to stress. Functional analysis in Mediterranean ecosystems. NATO, ASI Series, vol G15, Springer Verlag, Berlin, Heidelberg, New York

Tenhunen JD, Lange OL, Braun M (1981) Midday stomatal closure in mediterranean type slerophylls under simulated habitat conditions in an environmental chamber. II. Effects of the complex leaf temperature and air humidity on gaz exchange of Arbutus unedo and Quercus ilex. Oecologia 50, 5-11

Tenhunen JD, Lange OL, Harley PC, Beyschlag $W$, Meyer A (1985) Limitations due to water stress on leaf net photosynthesis of Quercus coccifera in the Portuguese evergreen scrub. Oecologia 67, 23-30

Tenhunen JD, Lange OL, Jahner D (1982) The control by atmospheric factors and water stress of midday stomatal closure in Arbutus unedo growing in natural macchia. Oecologia $55,1656-1669$

Vignes D (1988) Les échanges gazeux et leur régulation chez deux espèces de chênes à feuilles persistantes (Quercus ilex et Quercus suber). Bull Soc Bot Fr 135 Actual bot 1, 99108

William WE (1983) Optimal water use efficiency in a california shrub. Plant Cell Environ 6, 145-151 